\title{
Designing Laboratory Procedures to Enhance Graduate Attributes
}

\author{
Salim Ahmed, Syed Imtiaz, Lesley James and Darlene Spracklin-Reid \\ Faculty of Engineering \& Applied Science \\ Memorial University of Newfoundland \\ St. John's, NL A1B 3X5 \\ sahmed, simtiaz, ljames, darlenesr@mun.ca
}

\begin{abstract}
In undergraduate laboratory courses, students often perform experiments by following standard procedures designed by the instructors or outlined in the laboratory manuals. The calculation procedures are also provided to estimate certain parameters using experimental data. Finally they report the findings from the experiments. These activities lead to a number of different learning outcomes and students achieve a number of attributes. They gain experience with problem analysis, use of engineering tools, investigation and communication which are identified as required graduate attributes by the Canadian Engineering Accreditation Board. However, the important aspect of design that may be achieved through the development of structured experimental procedure remains unattended. In this article we discuss an approach for undergraduate laboratory experiments to enhance the attributes of engineering graduates. In this approach students are not provided with the experimental procedure; instead they need to develop the procedure to meet the goal of the experiments. This approach is currently being implemented in the laboratory component of a Process Dynamics and Control course at the memorial University of Newfoundland. A survey on students' achievement of graduate attributes as well as an assessment of learning outcomes will be carried out to evaluate the effectiveness of the approach.
\end{abstract}

\section{Introduction}

The importance of the laboratory courses in an engineering program is well recognized. According to [1] "Engineering without labs is a different discipline. If we cut out labs we might as well rename our degrees Applied Mathematics". However laboratory courses are expensive, messy, hard to teach, time consuming and not connected to the university's other mission - research [2]. Also the experience with the teaching of laboratory courses varies widely. As shared in [3] regarding laboratory exercises "our

CEEA13; Paper 133

Montreal, QC; June 17-20, 2013 approach can be described as using $20^{\text {th }}$ century technology to control $19^{\text {th }}$ century type processes in an $18^{\text {th }}$ century learning environment while creating engineers for the $21^{\text {st }}$ century". While in the same article the authors reported how the situation was overturned [3], there seems to be general lack of interest in part of the teaching community regarding renovation of the laboratory courses. This is reflected in the conferences on engineering education. Out of the 93 papers published in the proceedings of the 2102 CEEA conference, only about two were related to laboratory courses. The number is similar for the previous CEEA conferences. While laboratory courses are crucial for the success of future engineers, poorly designed and trivial laboratory experiments [4] may cause the students to loss interest in engineering practice. In this article the discussion is not on the development or utilization of resources related to laboratory courses. It is understandable that even the well reputed universities may not have proper laboratory equipment in sufficient numbers to meet the need of all students. However, even less equipped laboratories can help students in gaining higher level skills if the laboratory procedures are designed properly. The focus of this article is on how to design laboratory course to enhance learning outcomes and the graduate attributes.

Traditionally, in undergraduate laboratories, experiments are performed following a standard route; students are provided with the procedure, instructors demonstrate the operation of the equipment, students run the experiment with help from the teaching assistants and collect prescribed data. After finishing the experiment, students perform predefined calculations outlined in the procedure to obtain certain results identified in the objectives. Finally they document the findings from the experiments in the form of a report. These activities lead to a number of different learning outcomes and students achieve a number of attributes. In addition to becoming familiar with the operations of real processes, they achieve the attributes of problem analysis, use of engineering tools, investigation and communication which are

-1 of $6-$ 
identified as required graduate attributes by the Canadian Engineering Accreditation Board (CEAB). However, the important aspect of design that may be achieved through development of structured experimental procedure remains unattended. In this study we discuss a methodology for laboratory experiments to be performed by undergraduate students to enhance their design attribute as well as to achieve other attributes to higher levels. The distinguishing aspect of the approach is that the students will not be provided with the experimental procedure; instead they will be required to develop the procedure to meet the objectives of the experiments. The strategy will be applied in the laboratory component of a course titled "Process Dynamics and Control" covering topics on dynamic modeling and controller design. The course is offered to term 7 students in the Process Engineering program at the Memorial University of Newfoundland. Half of the students will perform one experiment following the traditional way while the other half will follow the proposed route. For a second experiment the groups will switch the approach. A survey will be carried out to extract response of the students regarding their attributes achieved by performing the experiments. Also an assessment of the learning outcomes from this exercise will be done. A comparative analysis of the level of achievement of the attributes will be carried out based on the assessment results and the response of the students through the survey. Although the number of expected response will be low, the study will result in a preliminary analysis of the effectiveness of the proposed route. We will continue the study beyond the current year and beyond the current course to exploit its benefits and extract statistically significant results to validate the outcome of the study.

The remainder of the article is structured as follows. The general purpose of laboratory courses and the graduate attributes that can be achieved through laboratory courses are discussed in section 2 . An unstructured way to perform laboratory experiments is outlined in section 3. The anticipated outcomes of the proposed approach are discussed in section 4. Means to demonstrate the effectiveness of the procedure is outlined in section 5. Some implementation issues are discussed in section 6 followed by the concluding remarks in section 7 .

\section{Purposes of a Laboratory Course}

The most important aspect of the design of a laboratory course is to define its purpose and to make the students aware of the purpose. It is worthwhile to

CEEA13; Paper 133

Montreal, QC; June 17-20, 2013 mention that the purpose of a laboratory experiment refers to the pedagogical intentions of the instructor i.e. the reasons why a particular laboratory activity is incorporated and how the activity is intended to result in planned student learning [5].

\subsection{Objectives of Experimental Works}

A laboratory course or the laboratory component of a course may many objectives. By doing experimental engineering works students can learn a variety of experimental skills including recording, analyzing and interpreting data. Through hands-on working students can observe and understand concepts which are otherwise abstract in some sense. They can learn to function in a real world environment and realize the effects of uncertainty and unseen factors. For students who understand a concept better by doing things compared to by reading or listening to lectures, laboratory works provide them an opportunity for higher level of understanding. Students also become familiar with modern engineering equipment, data acquisition accessories and control tools. Experimental works may also need the use of concepts learned in other courses which enhance their skills to apply knowledge from different sources. Students can also be trained on the aspects of personal and process safety during experiments. Finally experimental results may lead to discovery of previously unknown phenomenon in a process.

While the above list is attainable through experimental works, a single laboratory cannot meet all the objectives. The purpose of a laboratory course should be defined carefully to meet the program requirements and to complement graduate attributes. Only through proper definition of the purpose of an experimental work, the desired outcomes can be achieved.

\subsection{Gaining Graduate Attributes}

Most, if not all, of the twelve graduate attributes outlined by the CEAB that the graduates of an engineering program should possess, can be achieved through laboratory courses. A knowledge base for engineering can be enhanced by the observation of engineering phenomena through experimentation. Problem analysis skills can be developed by designing the experimental procedures to obtain necessary information to reach conclusions from experiments. The attribute of investigation can be achieved by analysis and interpretation of data and by synthesis of information to draw substantiated conclusions. Through design of experiments to meet the objectives 
of laboratory works, students can achieve the design attribute. In the laboratory, students use engineering tools for experimentation, data acquisition and analysis. Laboratory courses enhance the team work skills as experiments are typically done in teams. Students are required to present the results of experiments by submitting reports which enhance their communication skills. Through reporting properly and in a timely manner, students get the opportunity to demonstrate professionalism. Through observations of real process phenomena students realize the effects of engineering operations on safety and environment. The ability of the students to apply professional ethics, accountability and equity can be enhanced by sound practice of reporting the findings from experiments in a professional and ethical way. While students face the effect of uncertainty in real life through experiments, they realize the need for optimization from both process and optimization perspectives. Proper time and resource management skills can also be developed by properly planning laboratory works. Students may need to get information from sources other than their textbooks which enhance their skills to identify and address their educational needs and thus to enhance their life-long learning attribute.

It is understandable that no laboratory can be optimal for achieving all of the graduate attributes. Laboratory procedures can and should be designed and structured based on the program requirements to achieve the desired graduate attributes.

\section{An Unstructured Laboratory Procedure}

Laboratory exercise may be carried out in a structured or in an unstructured way [6]. Also a mixed approach can be taken to provide the students a guided experience. While all of these approaches have advantages as well as disadvantages, the structure of a laboratory should be defined based on the purpose of the course.

The laboratory experiments discussed in this article is part of the course titled "Process Dynamics and Control" offered to the students enrolled in the term 7 of the Process Engineering program at the Memorial university of Newfoundland. The Faculty of Engineering and Applied Science at Memorial offer a co-operative program where the students complete at least five work terms by the time they enroll in term 7 . Thus students registered in the course have a substantial real life experience and some of them get exposure to industrial control systems. With such a background of the students it may be more appropriate to conduct experiments in an unstructured or in other words open-ended way. Another motivation to adopt

CEEA13; Paper 133

Montreal, QC; June 17-20, 2013 an unstructured approach to experimentation comes for the purpose of enhancing the design attributes of the students which include design of solution procedures to solve open-ended problems. It is anticipated that the unstructured approach will help to achieve other graduate attributes to higher levels.

\subsection{The Experimental Set-ups}

The experimental set-ups for the process dynamics and control laboratory include two laboratory scale demonstration equipment; a level control equipment and a temperature control set-up. Both of the equipment are designed to apply single input single output controller. Two other set-ups to demonstrate multivariate control interaction and to apply multiple input multiple output controller is currently under commissioning. All these experimental set-ups are being connected to a Delta-V Distributed Control System (DCS).

\subsubsection{The level control equipment}

The level control trainer is Gunt's RT 512 unit equipped with industrial grade control systems. The trainer provides a comprehensive experimental introduction to the fundamentals of control engineering using an example of level control. The spectrum of experiments comprises the behaviour of control circuits, detection of step response, investigation of disturbances and optimization of control responses.

\subsubsection{The temperature control equipment}

The temperature control set-up is TecQuipment's TE3300/05 unit with a three speed pump to create flow around a temperature circuit. The pump transfers water from a reservoir through an in-line heater, a coiled delay pipe and a heat exchanger, before returning the water to the reservoir. A bypass valve allows the user to create disturbance. Three thermocouples measure temperatures in the water flow. A three position switch connects the selected thermocouple to a temperature transmitter. The controller output connects to a thyristor heater control circuit which controls the supply of electric power to the heater. A dedicated data acquisition system is in place for collecting and storing data.

\subsection{Experimental Procedures}

In the proposed unstructured approach, students are not provided with the procedure to carry out the experiments; instead, it is required that they design the 
experimental procedure, follow it during their laboratory experiments, identify and collect required data, outline calculation methods and carry out necessary calculations. The ultimate goal of the laboratory exercise is to design and implement controllers for the processes under consideration. Controller design involves two major steps: identification of a process model and estimation of the tuning parameters for the controller. These two steps will be carried out in two lab sessions. The laboratory experiments will be carried out in groups. Each group will spend two laboratory sessions for each experimental set-up. The approach is detailed below.

\subsubsection{Orientation and pre-lab}

Orientation sessions will be conducted by the instructor and the teaching assistants. At the beginning of the orientation sessions, students will be informed about the safety aspects of the laboratory and the required protection equipment for the laboratory. Then students will be given a brief introduction to each of the experimental set-ups. The manuals for the equipment that contains the information about the different components of the set-ups will be made available to the students ahead of time and students are required to familiarize themselves with the equipment manuals before coming to the orientation session. In an effort to help students in familiarizing with the equipment, some homework problems are designed based on the experimental set-ups. During the orientation session, students will be given information about the instruments e.g. the valves, the measurement devices e.g. the thermocouples and other auxiliary components of the set-ups to make them aware of the significant other elements in real plant operations. Detailed information about the associated software to run the experiments will also be provided during the orientation.

As pre-lab activities students will be required to study the equipment in details, define a control objective and select the proper control configuration to meet the desired objective. Based on the selected control configuration, they will be required to design the identification experiment to obtain a model for the process. The experimental procedure should identify the information and data required to get the desired model; also it should contain details about the step-bystep procedure to run the experiment to get the desired data. Finally, the procedure to get the model from the experimental data should also be documented with proper justification for the choice of the calculation methods. These pre-lab activities should be documented and submitted at the beginning of the laboratory experiment session.

CEEA13; Paper 133

Montreal, QC; June 17-20, 2013

\subsubsection{The identification exercise}

During the laboratory exercise for model identification, students will collect necessary and sufficient data to get a satisfactory model following the designed experimental procedure. The data file should be submitted electronically at the end of the exercise. Through visual observation of the data students will have to decide whether the collected data meet the requirements.

\subsubsection{Post-lab to the identification exercise}

As the post-lab activity to the identification exercise students will be required to choose a proper algorithm to get the process model from the collected data set, perform necessary calculations to get the model and finally prepare a report on the identification exercise and submit it.

\subsubsection{Pre-lab to controller implementation}

As mentioned earlier, each experiment will require two laboratory sessions. In the second session, a controller will be implemented to the process and the performance of the controller will be evaluated. As a pre-lab to the exercise on controller implementation, students will be required to design the controller using the identified model and a suitable controller design algorithm. Proper justification for the choice of the algorithm should be provided. Also students will be required to identify a criterion for the evaluation of the performance of the controller. Finally they should outline the procedure to implement the controllers. The information and data required to collect during the controller implementation exercise should also be identified. The calculations, the selected control performance evaluation criterion and the experimental procedure should be documented and submitted as a report at the beginning of the second lab session.

\subsubsection{Controller implementation exercise}

During the second lab session students will implement the designed controller, run the process and collect necessary data to evaluate the performance of the controller. From a preliminary evaluation of the controller performance, students may need to fine-tune the controller and conduct additional run to obtain more information about the performance of the controller.

\subsubsection{Post-lab to controller implementation}

As the final step of the laboratory exercise, students will be required to evaluate the performance of the -4 of 6 - 
designed controllers based on the selected criterion and suggest possible fine-tuning of the controller parameters to get a better response of the process under controlled conditions. Finally the results on the performance of the controller should be documented as a report and submitted.

\section{Anticipated outcomes of the proposed approach}

\subsection{Enhancing Student Engagement}

Students often come to the laboratory less prepared than necessary to perform a laboratory exercise and to benefit from doing the experiments. There have been efforts to administer pre-lab quizzes to make sure that students are familiar with the experimental procedure and methodology. While this motivates some students to study the laboratory procedures and manuals in advance, students rely on the supplied procedure to perform the experiment. If the students themselves are required to design the experimental procedure, they may find themselves more engaged during the laboratory sessions. Also they will have to study the process in details to design the procedure. In addition, in real life problems, professional engineers are not provided with procedures to solve problems; they need to design procedures to solve problems. Designing the experimental procedure will provide the students an opportunity to perform workplace style learning exercise.

\subsection{Enhancement of Graduate Attributes}

It is anticipated that the unstructured approach to the laboratory exercise will enhance a number of graduate attributes.

\subsubsection{Problem analysis}

Design of the experimental procedure will require that each of its steps be justified to avoid unnecessary actions during experiments. At the same time students will have to ensure that they do not miss a necessary step. In addition, they will have to identify data and information to be collected during the exercise. All these require detailed analysis of the processes as well as the associated control mechanisms. Thus it is anticipated that the problem analysis skill of the students will be developed better by following the unstructured approach compared to the structured approach where experimental procedures are provided by the instructors.

CEEA13; Paper 133

Montreal, QC; June 17-20, 2013

\subsubsection{Design}

Developing the procedure to conduct experiments will enhance the design skills of the students. The open-ended nature of the problem is suitable for sharpening the design skills and it is anticipated that performing the experiments in an unstructured way will help students in understanding the concepts of design.

\subsubsection{Team work and communication}

Team work and the communication are the two main graduate attributes achieved extensively through laboratory courses. The unstructured approach will require more pre- and post-lab activities to be performed by the team. To come up with a procedure to conduct the experiments will require effective discussions among the team members. The open ended nature of the exercise may foster debates for a consensus. This will enhance both the team work skills and the communication skills of the students.

In addition to the enhancement of the above mentioned graduate attributes, it is anticipated that the unstructured approach to the laboratory experiment will provide better knowledge about the corresponding processes and the associated engineering phenomena. It will be helpful to develop the investigation skills as well. The workplace style learning environment will provide the opportunity to realize their professional responsibilities. The experimental procedure will be checked by the instructor to make sure that none of the steps leads to an unsafe condition in the laboratory. Students will be notified in advance that they will be penalized for failing to design the experiment to run in a safe manner. This will enhance their ability to realize the impacts of engineering design. Finally by structuring the experimental activities into time-bound step wise tasks, the project management skills of the students will be enhanced.

\section{Evaluation Methodologies}

It is anticipated that the unstructured approach will enhance graduate attributes as well as enhance the engagement of the students during experiments. To verify that indeed the expected benefits of the proposed approach are obtained, laboratory experiments will also be performed in a structured or the so called cookbook-type approach. Students will perform the design and implement the controller for one experimental set-up using the proposed approach; while for the other set-up, they will take the cookbook approach where the experimental procedures will be

-5 of $6-$ 
provided by the instructor. A survey will be carried out to collect feedback from the students on their learning and the achievement of graduate attributes. An assessment of the learning outcomes will be done in the form of laboratory quizzes to assess the extent of learning outcomes. A comparative study of the two approaches will be done based on the results from the survey and the assessment.

\section{Implementation Issues}

\subsection{Scheduling of Experiments}

The identification exercise will be carried out during the first half of the term from the third to the sixth week. Half of the students will perform identification experiments using the level control setup while the other half will use the temperature control set-up. After the first experiment by all groups, they will switch the set-up; also they will perform the second identification experiment following the structured way with the experimental procedures provided by the instructor. The structured approach will be practiced after the unstructured approach is completed to make sure that students do not have access to a designed procedure during the unstructured approach. The same sequence will be followed for the controller implementation experiments during the second half of the term.

\subsection{Workload of the Students}

Additional workload for the student will result due to the extensive pre-lab activities to design the experimental procedures. To compensate for the additional workload some homework problems will be designed based on the experimental set-ups. This, in one hand, will help the students to earn some extra credits from their laboratory works; on the other hand, it will make the laboratory exercises more related to the theoretical part of the course. In addition, it will bring some diversity in the homework.

\subsection{Ethical Aspects}

Involving students in pedagogical experiments always raises some ethical questions. The issue becomes more pressing when different groups of students are exposed to different learning environments. In this study, all of the students will follow both the structured and unstructured approach for experimental activities, however, in turns. The only difference is in the experimental set-ups used by different groups of students for the two approaches. While half of the students will use the temperature control set-up for the unstructured approach, the other half will use the level control set-up. The similarity in the two set-ups ensures that the learning environment and tools remain comparable.

\section{Concluding Remarks}

Laboratory courses are resource intensive both for the academic programs and for the students. It is costly to establish and maintain a laboratory. It requires significant amount of time from technical staffs, teaching assistants as well as from instructors to run a laboratory course. The learning outcomes from laboratory works may not be proportional to the resources utilized if the course is not well managed. Even for smoothly running laboratory courses there may be significant opportunities to enhance the learning outcomes. In this article we outline an unstructured approach to laboratory courses to enhance graduate attributes. This approach is not a new finding; however, adopting the approach for the purpose of enhancing graduate attributes may be considered to be novel and fruitful. We came forward to share our experience in the early stage of this study to engage in discussions and debates on laboratory courses. We believe that through collaborative efforts from different universities, laboratory courses can be significantly improved to meet the desired attributes of future graduates.

\section{References}

[1] C. Eastlake, "Tell me, I'll forget; show me, I'll remember; involve me, I'll understand (The tangible benefits of labs in the undergraduate curriculum)". Proc. ASEE Annual Conf., Washigton DC, 1986.

[2] P. Wankat, F. Oreovicz, Teaching Engineering, McGraw-Hill, Inc., 1993.

[3] G. Norval, P. Szabo, G. Wilson, P. Jowlabar, "A new approach to the unit operations laboratory at the University of Toronto". Proc. 2011 CEEA Conf., St. John's, NL, June 2011.

[4] V. Skormin, V. Nikulin, "Engineering Laboratory Accessible via the Internet", Proc. 2001 ASEE Conf., Albuquerque, New Mexico, June 2001.

[5] C. Hart et al. "What is the purpose of this experiment? Or Can students learn something from doing experiments?" J. of Research in Science Teaching, 37(7) pp 655-675, 2000.

[6] L. Alexander, R. Davis, K. Azima, "The Laboratory" Guides for improvement of instructions in higher education, No. 9, Michigan State Univ., MI, 1978.

-6 of $6-$ 